\title{
ON COMMUTING ELEMENTS AND EMBEDDINGS OF GRAPH GROUPS AND MONOIDS
}

\author{
Mark Kambites \\ Fachbereich Mathematik / Informatik, Universität Kassel \\ 34109 Kassel, Germany \\ kambites@theory.informatik.uni-kassel.de
}

\begin{abstract}
We study commutation properties of subsets of right-angled Artin groups and trace monoids. We show that if $\Gamma$ is any graph not containing a four-cycle without chords, then the group $G(\Gamma)$ does not contain four elements whose commutation graph is a four-cycle; a consequence is that $G(\Gamma)$ does not have a subgroup isomorphic to a direct product of non-abelian groups. We also obtain corresponding and more general results in the monoid case.
\end{abstract}

\section{INTRODUCTION}

Much research has centred upon finitely generated monoids and groups defined by presentations in which the only relations are commutators of certain of the generators. Monoids of this type, which are variously called graph monoids, trace monoids and free partially commutative monoids, arise naturally in the theory of computation, where they form a natural model of concurrent processing [8, 9]. Graph groups can be used to model concurrent processing with invertible operations; they also play an important role in combinatorial group theory, where they are usually known as right-angled Artin groups [2, 3, 6, 13].

The subgroup structure of graph groups has been extensively studied, with extremely interesting results (see, for example, 2, 7, 10]). Likewise, there is considerable interest in submonoids of trace monoids. Of particular importance in computer science are those submonoids of trace monoids which are themselves trace monoids; an embedding of a trace monoid into another is called a trace coding [8, 17, since it is the natural partially commutative analogue of a word coding. Trace codings have been extensively studied, with particular attention paid to decidability questions [4] 5].

Closely related to possible embeddings of graph groups or monoids, are the possible commutation properties of subsets (and multisubsets) of groups and monoids. These have been studied, in the group case, by Duncan, Kazatchkov and Remeslennikov [12. Motivated by considerations from algebraic geometry over groups, they associated to each finite graph $\Gamma$ the class of groups which admit elements whose commutation properties are described by the graph $\Gamma$.

In this paper, we consider the commutation properties of subsets of both graph groups and graph monoids. In particular, we study certain key graphs $\Gamma$ which have the property that a graph group $G(\Omega)$ or graph monoid $M(\Omega)$ admits a subset whose commutation properties are described by $\Gamma$ only when $\Omega$ contains an embedded copy of $\Gamma$. As a consequence, we obtain some negative results regarding embeddings of both graph groups and graph monoids. 
In addition to this introduction, this paper comprises four sections. We begin, in Section 2 by briefly introducing graph groups and monoids, along with the notation and foundational results which we shall need in the following sections.

Section 3 is devoted to graph groups. We show that a graph group admits a subset whose commutation graph is a four-cycle if and only if it contains an embedded (without chords) four-cycle. A consequence is that a graph group contains a direct product of non-abelian free groups as a subgroup only when its graph contains a four-cycle. This fact, which was conjectured by Batty and Goda 1], is of particular interest because of the many properties which are shared by free groups and free abelian groups but not by direct products of free groups. One example is decidability of the algorithmic subgroup membership problem. A construction of Mikhailova [15. 16] shows that this problem is undecidable for direct products of free groups, and hence for any graph group $G(\Gamma)$ where $\Gamma$ contains a chord-free four-cycle. On the other hand, a recent result of Kapovich, Weidmann and Myasnikov [14 shows that the problem is decidable for $G(\Gamma)$ when $\Gamma$ contain no chord-free cycles. There remains the case of groups $G(\Gamma)$ where $\Gamma$ contains chord-free cycles but not of length four. Our result shows that Mikhailova's construction does not present an obstruction to decidability of subgroup membership in these groups.

In Section 4 we turn our attention to graph monoids. We show that for certain graphs $\Gamma$, a graph monoid admits a subset with commutation graph $\Gamma$ if and only if its graph contains an embedded copy of $\Gamma$. As a consequence, we deduce a related restriction on embeddings of direct products of free monoids.

In Section [5] ask what other graph groups and monoids have similar properties. It transpires that the monoid results from Section 4 are best possible, in the sense that every graph monoid which is not a direct product of free monoids of rank 1 and 2 admits an embedding into a graph monoid without a corresponding embedding of graphs. This contrasts with the group case, where it follows from a result of Droms, Servatius and Servatius [1] that the graph group on the three-edge line does not embed into a graph group without a corresponding embedding of graphs. Finally, we give a combinatorial construction which embeds any member of a large class of graph groups into another graph group, without a corresponding embedding of graphs.

\section{Graphs, Monoids And Groups}

In this section, we briefly introduce the concepts, notation and foundational results which will be required in the sections that follow. We concentrate here on such of the theory as is common to the monoid and group cases; ideas which are particular to groups or monoids will be introduced in Sections 3 and 4 respectively.

2.1. Graphs. By a graph $\Gamma$ we mean a mean a set $V(\Gamma)$ of vertices together with a reflexive, symmetric relation $E(\Gamma) \subseteq V(\Gamma) \times V(\Gamma)$. Two vertices $u, v \in V(\Gamma)$ are adjacent if $(u, v) \in E(\Gamma)$. The degree $|\Gamma|$ of $\Gamma$ is the cardinality of $V(\Gamma)$. The degree of a vertex $v$ in $\Gamma$, denoted $|v|_{\Gamma}$, is the number of vertices adjacent to and distinct from $v$.

A morphism from a graph $\Gamma$ to a graph $\Omega$ is a map from the vertex set of $\Gamma$ to that of $\Omega$ which preserves adjacency (but not in general non-adjacency). An embedding of graphs is a morphism which is injective on vertices and which preserves nonadjacency. If $S$ is a subset of $V(\Gamma)$ then the subgraph of $\Gamma$ induced by $S$ is the graph with vertex set $S$ and edge set $E(\Gamma) \cap(S \times S)$. 


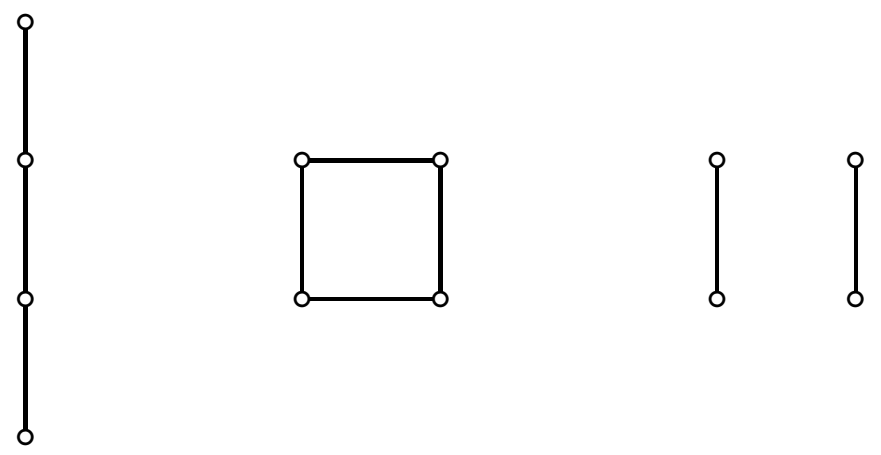

Figure 1 . The graphs $L_{3}=\overline{L_{3}}, C_{4}$ and $\overline{C_{4}}=E_{0,2}$.

A (connected) component of a graph is a maximal set of vertices such that every pair of vertices contained is connected by a path. A graph is connected if it has only one component, and disconnected otherwise.

Let $\Gamma$ and $\Omega$ be graphs with disjoint vertex sets. Then the connected product $\Gamma \times \Omega$ is the graph with vertex set $V(\Gamma \times \Omega)=V(\Gamma) \cup V(\Omega)$ and edge set

$$
E(\Gamma \times \Omega)=E(\Gamma) \cup E(\Omega) \cup(V(\Gamma) \times V(\Omega)) \cup(V(\Omega) \times V(\Gamma)) .
$$

The complement $\bar{\Gamma}$ of $\Gamma$ is the graph with the same vertex set as $\Gamma$, and in which two distinct vertices are adjacent exactly if they are not adjacent in $\Gamma$. A (co-connected) co-component of $\Gamma$ is a component of $\bar{\Gamma}$; the graph $\Gamma$ is called coconnected or co-disconnected if $\bar{\Gamma}$ is connected or disconnected respectively;

Figure 1 shows three examples of graphs which are important in the study of graph monoids and groups; they are the three-edge line $L_{3}$, the four-cycle (or "square") $C_{4}$ and its complement $\overline{C_{4}}$. For clarity, we draw the graphs without the loops at the vertices. Note that $\overline{L_{3}}$ is isomorphic to $L_{3}$ - we say that that $L_{3}$ is self-complementary. The complement graph $\overline{C_{4}}$ has two connected components, each of which consists of two vertices joined by an edge. More generally, for $i, j \geq 0$ we denote by $E_{i, j}$ the unique graph with $i$ vertices of degree 0 and $2 j$ vertices of degree 1 , so that $\overline{C_{4}}=E_{0,2}$. Another example which will be important for us is the unique two-vertex disconnected graph $\overline{E_{0,1}}=E_{2,0}$.

2.2. Graph Monoids and Groups. Let $\Gamma$ be a graph. The graph monoid $M(\Gamma)$ and graph group $G(\Gamma)$ are the monoid and group respectively defined by the presentation

$$
\langle V(\Gamma)| a b=b a \text { for all }(a, b) \in E(\Gamma)\rangle .
$$

There is an obvious embedding of $M(\Gamma)$ into $G(\Gamma)$, and it is often convenient to regard the former as a submonoid of the latter. It is also frequently useful to consider a set of monoid generators for $G(\Gamma)$. With this in mind, we let

$$
U(\Gamma)=\left\{a, a^{-1} \mid a \in V(\Gamma)\right\}
$$

be a symmetrised set of generators for $G(\Gamma)$. If $u \in U(\Gamma)^{*}$ then we denote by $\bar{u}$ the element of $G(\Gamma)$ (and hence, where appropriate, $M(\Gamma)$ ) represented. 
Returning to our examples of graphs from above, it is easily seen that $G\left(C_{4}\right)$ [respectively, $\left.M\left(C_{4}\right)\right]$ is isomorphic to a direct product two free groups [monoids] of rank 2. More generally, $G\left(\overline{E_{i, j}}\right)\left[M\left(\overline{E_{i, j}}\right)\right]$ is a direct product of $i$ free groups [monoids] of rank 1 and $j$ free groups [monoids] of rank 2. On the other hand, $G\left(L_{3}\right)\left[M\left(L_{3}\right)\right]$ is both freely and directly indecomposable, and hence cannot be built up from free groups [monoids] using only the operations of free and direct product. In fact, $L_{3}$ is known to be the minimum graph with the latter property, not only in terms of number of vertices, but also with respect to embedding [11.

The length of an element $g \in G(\Gamma)$ is the minimum length of a word in the $U(\Gamma)^{*}$ representing $g$; a word of this length which represents $g$ is called a reduced word for $g$. A reduced factorisation for $g$ is an expression $g=g_{1} \ldots g_{n}$ where the sum length of the $g_{i}$ s equals the length of $g$. A prefix [suffix] of $g$ is an element $h \in G(\Gamma)$ which is the first factor [last factor] in some reduced factorisation for $g$.

The support of $g$ is the set of all vertices $t \in V(\Gamma)$ such that either $t$ or $t^{-1}$ or both occur in any (and hence in every) reduced word for $g$. We write $t \in g$ to denote that $t \in V(\Gamma)$ lies in the support of $g \in G(\Gamma)$. We say that two elements $u, v \in G(\Gamma)$ commute totally if every generator in the support of $u$ commutes with every generator in the support of $v$. A generator $a \in U(\Gamma)$ is called central if $a$ has degree $|\Gamma|-1$, that is, if $a$ represents a central element in $G(\Gamma)$.

All of the definitions above apply by restriction to elements of $M(\Gamma)$.

2.3. Commutation Graphs. Given a subset $S$ of a group $G$, the commutation graph of $S$ (in $G$ ) is the graph with vertex set $S$, and an edge joining two vertices exactly exactly if they commute in $G$.

Let $G$ be a group and $\Omega$ a graph. Following [12, we say that a group $G$ satisfies $\phi(\Omega)$ if there exists a function $\sigma: V(\Omega) \rightarrow G$ with the property that $u, v \in V(\Omega)$ are adjacent if and only if $\sigma(u)$ and $\sigma(v)$ commute. Since $\sigma$ need not be injective, this is in general slightly weaker than saying that $G$ has a subset with commutation graph $\Omega$. However, with $\Omega$ countable and $G$ torsion-free the two notions are easily seen to coincide, and it is this case which will be of interest to us.

\section{Graph Groups and $C_{4}$}

Our main aim in this section is to show that a graph group $G(\Gamma)$ does not satisfy $\phi\left(C_{4}\right)$ unless $\Gamma$ contains an embedded copy of $C_{4}$. In particular, it follows $G(\Gamma)$ admits a subgroup isomorphic to a direct product of free groups if and only if $\Gamma$ contains an embedded copy of $C_{4}$; this proves part of a conjecture of Batty and Goda 11. In Section 4] we shall prove an even stronger result in the monoid case.

Our main proof makes use of a theorem of Servatius [18, characterising centralizers of elements in graph groups; we begin by briefly recalling some terminology and results from his paper. An element $e \in G(\Gamma)$ is called cyclicly reduced if it is of minimal length amongst elements in its conjugacy class. It is easily seen that every element of $G(\Gamma)$ can be written uniquely as a reduced product $g=p h p^{-1}$ where $h$ is cyclicly reduced. The element $h$ is called the cyclic reduction of $e$.

Now suppose $h$ is cyclicly reduced; and let $\Omega$ be the subgraph of $\Gamma$ induced by the support of $h$. It is straightforward to show that we can write $h$ in the form $h_{1}^{i_{1}} h_{2}^{i_{2}} \ldots h_{n}^{i_{n}}$ where each $i_{j}$ is positive, each $h_{j}$ has support contained in different coconnected component of $\Omega$, and no $h_{j}$ is a proper power. Moreover, this expression is unique up reordering of the factors. The elements $h_{i}$ and their inverses are called the pure factors of $h$. Notice that the pure factors commute with one another. 
Theorem 1 ((The Centralizer Theorem, Servatius 1989 [18])). Suppose $g=p h p^{-1}$ reduced, with $h$ cyclicly reduced. Then $k$ commutes with $g$ if and only if $k$ can be written as $p k_{1} k_{2} p^{-1}$ where $k_{1}$ is a product of pure factors of $h$, and $k_{2}$ commutes totally with $h$.

The following combinatorial observation is probably well-known.

Proposition 2. Let $u, v \in G(\Gamma)$. Then there exist reduced factorisations $u=u^{\prime} x$ and $v=x^{-1} v^{\prime}$ such $u^{\prime} v^{\prime}$ is a reduced factorisation for $u v$.

Proof. Suppose false for a contradiction, and let $u$ and $v$ be counterexamples of minimal total length. Certainly $u v$ is not a reduced product, or setting $u^{\prime}=u$, $v^{\prime}=v$ and $x=1$ would give the required properties.

Now let $\tilde{u}$ and $\tilde{v}$ be reduced words representing $u$ and $v$ respectively. Since $u v$ is not reduced, we can write $\tilde{u} \tilde{v}=\tilde{a} t \tilde{b} t^{-1} \tilde{c}$ for some (positive or negative) generator $t$ and words $\tilde{a}, \tilde{b}$ and $\tilde{c}$ such that $t$ commutes totally with $\tilde{b}$.

The factor $t \tilde{b} t^{-1}$ cannot be contained entirely in $\tilde{u}$ or $\tilde{v}$, since these are reduced words. It follows that the initial $t$ must lie in $\tilde{u}$, and commute with every letter which comes after it in $\tilde{u}$. By commuting it to the end, we may assume without loss of generality that $t$ is the last letter in $\tilde{u}$. By a symmetrical argument, we may assume also that $t^{-1}$ is the first letter in $\tilde{v}$.

Write $\tilde{u}=\tilde{a} t$ and $\tilde{v}=t^{-1} \tilde{b}$, and let $a$ and $b$ be the elements represented by $\tilde{a}$ and $\tilde{b}$ respectively. Now by the minimality assumption, there are reduced factorisations $a=u^{\prime} y$ and $b=y^{-1} v^{\prime}$ such that $u^{\prime} v^{\prime}$ is a reduced factorisation for $a b=u v$. Now set $x=y t$ to give $u=u^{\prime} x$ and $v=x^{-1} v^{\prime}$ as required.

We shall need a number of other preliminary results. The first two are of a rather technical nature.

Proposition 3. Suppose $y, t \in V(\Gamma)$ are two non-commuting vertices. Suppose $t \notin u w v$ where $y \notin u$ and $y \notin v$ but $w$ is represented by a reduced word beginning and ending with a positive or negative occurrence of $y$. Then $t \notin u$ and $t \notin v$.

Proof. Suppose false for a contradiction, and let $u$ and $v$ be elements of minimal total length such that the proposition fails, that is, such that $t \in u$ or $t \in v$. By left-right symmetry, we can assume without loss of generality that $t \in u$.

Let $\tilde{u}, \tilde{w}$ and $\tilde{v}$ be reduced words for $u, w$ and $v$ respectively, where $\tilde{w}$ begins and ends with a positive or negative occurrence of $y$. Certainly $\tilde{u} \tilde{w} \tilde{v}$ is reducible, or we would have $t \in u w v$, giving the required contradiction. Hence, there must exist a factorisation $\tilde{u} \tilde{w} \tilde{v}=\tilde{a} x \tilde{b} x^{-1} \tilde{c}$ where $\tilde{b}$ represents an element which commutes totally with the positive or negative generator $x$. Since the words $\tilde{u}, \tilde{v}$ and $\tilde{w}$ are reduced, the factor $x \tilde{b} x^{-1}$ cannot lie wholly in any one of those words. Thus, this factor must contain one end of $\tilde{w}$, and hence must contain $y$ or $y^{-1}$. Since $y \neq t$ and $y$ does not commute with $t$, it follows that $x \neq t$ and $x \neq t^{-1}$. Since at least one occurrence of $x$ must lie in $\tilde{u}$ or $\tilde{v}$, we know also that $x \neq y$ and $x \neq y^{-1}$

Now we can write $\tilde{a} \tilde{b} \tilde{c}=u^{\prime} w^{\prime} v^{\prime}$ where $u^{\prime}, w^{\prime}$ and $v^{\prime}$ are reduced scattered subwords of $\tilde{u}, \tilde{w}$ and $\tilde{v}$ obtained by deleting only occurrences of $x$ and $x^{-1}$, and the combined length of $u^{\prime}$ and $v^{\prime}$ is strictly less than that of $u$ and $v$. Moreover, it is clear that $w^{\prime}$ still begins and ends with a positive or negative occurrence of $y$. Hence, by the minimality assumption, it follows that $t \notin u^{\prime}$ and $t \notin v^{\prime}$, and hence that $t \notin \tilde{u}$ and $t \notin \tilde{v}$. Thus, $t \notin u$ and $t \notin v$, as required. 
Lemma 4. Let $p \in G(\Gamma)$ and $t \in V(\Gamma)$, and suppose $r \in G(\Gamma)$ is of minimal length such that $p$ has a reduced factorisation of the form qtr or $q t^{-1} r$. Suppose $c \in G(\Gamma)$ is such that $t \notin p c p^{-1}$ and the support of $c$ contains a generator which does not commute with $t$, and does not occur in the support of $p$. Then $c$ has a reduced factorisation of the form $c=r^{-1} t^{-1} d t r$ (if $p=q t r$ ) or $c=r^{-1} t d t^{-1} r$ (if $\left.p=q t^{-1} r\right)$.

Proof. We treat the case in which $p=q t r$; an entirely similar argument applies when $p=q t^{-1} r$.

Let $y \in c$ be a generator which does not commute with $t$ and does not occur in $p$. Let $\tilde{c}$ be a reduced word for $c$, and write $\tilde{c}=\tilde{u} \tilde{w} \tilde{v}$ where $y \notin \tilde{u}, y \notin \tilde{v}$ but $\tilde{w}$ begins and ends with a positive or negative occurrence of $y$. Let $u, w$ and $v$ be the elements represented by $\tilde{u}, \tilde{w}$ and $\tilde{v}$ respectively.

Now $t \notin p c p^{-1}=p u w v p^{-1}$, so applying Proposition 3 we see that $t \notin p u=q t r u$ and $t \notin v p^{-1}=v r^{-1} t^{-1} q^{-1}$.

Now by Proposition 2] there exist reduced factorisation s $p=p^{\prime} x$ and $u=x^{-1} u^{\prime}$ such that $p^{\prime} u^{\prime}$ is a reduced factorisation for $p u$. Now $t \notin p u=p^{\prime} u^{\prime}$, so clearly $p^{\prime}$ does not contain $t$. But $p^{\prime} x=p=q t r$, so it follows easily from the minimality assumption on $r$ that $x$ has a suffix $t r$, and hence that $u$ has a prefix $r^{-1} t^{-1}$ as required. A symmetrical argument shows that $v$ has a suffix $t r$.

Lemma 5. Suppose $\Gamma$ does not contain an induced copy of $C_{4}$. Suppose further that $G(\Gamma)$ has a subset $S$ with commutation graph isomorphic to $C_{4}$, one of whose elements a is cyclicly reduced. Then a commutes totally with itself and with those other members of $S$ with which it commutes.

Proof. Suppose $\{a, b, c, d\} \subseteq G(\Gamma)$ has commutation graph isomorphic to $C_{4}$, where $a$ fails to commute with $c$, and $b$ fails to commute with $d$. Suppose further that $a$ is cyclicly reduced. Let $a_{1}, \ldots, a_{n}$ be the pure factors of $a$. Then by Theorem 1 we can write $b=b_{1} b_{2}$ and $d=d_{1} d_{2}$ where $b_{1}$ and $d_{1}$ are products of pure factors of $a$, and $b_{2}$ and $d_{2}$ commute totally with $a$.

Notice that $b_{1}$ and $d_{1}$ commute, and both $b_{1}$ and $d_{1}$ commute totally with both $b_{2}$ and $d_{2}$. Now if $b_{2}$ commuted with $d_{2}$ then $b$ would commute with $d$, giving a contradiction. Hence, $b_{2}$ does not commute with $d_{2}$. It follows that some vertex $x \in b_{2}$ fails to commute with some vertex in $y \in d_{2}$. Now $x$ and $y$ commute with every vertex in the support of $a$, so if two vertices in the support of $a$ failed to commute then we would obtain a four-cycle in $\Gamma$, giving a contradiction. Thus, $a$ must commute totally with itself.

Now since the support of $b_{1}$ is contained in that of $a, b_{1}$ commutes totally with $a$. We already know that $b_{2}$ commutes totally with $a$, so it follows that $b$ commutes totally with $a$. By symmetry of assumption, $d$ also commutes totally with $a$, as required.

Lemma 6. Suppose $\Gamma$ does not have an induced subgraph isomorphic to the $C_{4}$ but $G(\Gamma)$ does satisfy $\phi\left(C_{4}\right)$. Then $\Gamma$ has a subset $S$ with commutation graph isomorphic to $C_{4}$, in which two commuting elements are cyclicly reduced.

Proof. Suppose $\{a, b, c, d\} \in G(\Gamma)$ has commutation graph isomorphic to $C_{4}$, where $a$ fails to commute with $c$, and $b$ fails to commute with $d$. Clearly, by conjugating the entire set, we may assume that one of these elements, say $a$, is cyclicly reduced. Now by Lemma 5 commutes totally with $a$. 
Now suppose $b=p^{-1} b^{\prime} p$ is a reduced factorisation where $b^{\prime}$ is cyclicly reduced. Clearly, the set $\left\{p a p^{-1}, p b p^{-1}, p c p^{-1}, p d p^{-1}\right\}$ also has commutation graph isomorphic to $C_{4}$. But the support of $p$ is contained in that of $b$, and hence $p$ commutes totally with $a$. It follows that $p a p^{-1}=a$ and $p b p^{-1}=b^{\prime}$ are both cyclicly reduced as required.

Theorem 7. Let $\Gamma$ be a graph containing no induced copy of $C_{4}$. Then $G(\Gamma)$ does not satisfy $\phi\left(C_{4}\right)$.

Proof. Suppose for a contradiction that $G(\Gamma)$ satisfies $\phi\left(C_{4}\right)$. By Lemma 6] there is a subset $\{a, b, c, d\} \subseteq G(\Gamma)$ with commutation graph isomorphic to $C_{4}$ with $a$ and $b$ commuting and cyclicly reduced. By Lemma [5 we may assume without loss of generality that $a$ and $b$ commute totally with themselves, with each other, and with $d$ and $c$ respectively.

Suppose $d=p^{-1} e p$ reduced with $e$ cyclicly reduced. Then $c$ commutes with $d$ so by Theorem 1 we can write $c=p^{-1} c_{1} c_{2} p$ where $c_{1}$ is a product of pure factors of $e$, and $c_{2}$ commutes totally with $e$.

Since $c$ does not commute with $a$, there must exist a letter $x \in a$ which fails to commute with a letter $y \in c$. Now $y$ must be in the support of at least one of $c_{1}$, $c_{2}$ and $p$. If $y \in c_{1}$ or $y \in p$ then $y \in d$; but $d$ commutes totally with $a$, so this contradicts the assumption that $x$ and $y$ do not commute. Thus, we must have $y \in c_{2}$.

Since $b$ and $d$ do not commute, there are non-commuting vertices $s \in b$ and $t \in d$. We know that $s$ and $t$ both commute with $x$, and that $s$ commutes with $y$. We know also that $s$ and $t$ do not commute, and that $x$ and $y$ do not commute. Since the graph is assumed to contain no induced copy of $C_{4}$, it must be that $y$ does not commute with $t$. Since $y \in c_{2}$ and $c_{2}$ commutes totally with $e$, we have $t \notin e$. But $t \in d=p e p^{-1}$, so we must have $t \in p$. However, since $t$ does not commute with $s \in b$, and $c$ commutes totally with $b$, we must have $t \notin c=p c_{1} c_{2} p^{-1}$.

Choose a reduced factorisation $q t r$ or $q t^{-1} r$ for $p$ such that $r$ has minimal length. We have already observed that $y \in c_{1} c_{2}$ does not commute with $t$, and certainly $y \notin p$ or we would have $y \in d$ and $y$ would have to commute with $x$. Applying Lemma 4 we see that $c_{1} c_{2}$ has a reduced factorisation of the form $r^{-1} t^{-1} f t r$. In particular, the support of $t r$ is contained in the support of $c_{1} c_{2}$.

Let $\Omega$ be the subgraph of $\Gamma$ induced by the support of $c_{1} c_{2}$. Notice that every vertex in the support of $c_{1}$ is connected to every vertex in the support of $c_{2}$. Thus, the support of $c_{1}$ and the support of $c_{2}$ are unions of disjoint sets of co-connected components of $\Omega$.

Now we claim that the support of $t r$ is co-connected in $\Omega$. Indeed, if not, then $r$ would contain letters from a co-component not containing $t$; it would follow that we could commute these letters back through $t$, contradicting the assumption that $r$ is of minimal length. It follows that the support of $t r$ lies in a single co-connected component of $\Omega$. In particular, the support of $t r$ is contained either in the support of $c_{1}$, or in the support of $c_{2}$.

But $t$ cannot be in the support of $c_{1}$, since $c_{1}$ is a product of pure factors of $e$, and $t$ is not in the support of $e$. On the other hand, the final letter of $t r$ cannot be in the support of $c_{2}$, since then it would commute with every letter in $e$, contradicting the assumption that $p e p^{-1}=q t r e r^{-1} t^{-1} q^{-1}$ is a reduced factorisation. This completes the proof. 
As an immediate corollary, we obtain a restriction on the graph groups which contain a subgroup or submonoid isomorphic to a direct product of non-abelian groups (or monoids).

Corollary 8. Let $\Gamma$ be a graph not containing an embedded copy of $C_{4}$. Then $G(\Gamma)$ has no subgroup [submonoid] isomorphic to direct product of 2 or more non-abelian groups [monoids].

Corollary 9. If $\Gamma$ does not contain an embedded copy of $C_{4}$, then $G(\Gamma)$ does not contain an embedded copy of $G\left(C_{4}\right)$.

\section{Graph Monoids And $\overline{E_{i, j}}$}

In this section, we show that if $\Gamma$ is a graph in which every vertex has degree $|\Gamma|-2$, that is, a graph of the form $\overline{E_{0, j}}$, then a graph monoid $M(\Omega)$ satisfies $\phi(\Gamma)$ only when $\Gamma$ embeds in $\Omega$. We deduce also that a direct product of (abelian and non-abelian) free monoids does not embed into a graph monoid without a corresponding embedding of graphs.

We recall some standard definitions from the theory of graph monoids. Given a graph $\Gamma$, we define a number of morphisms from $M(\Gamma)$ to free monoids of rank 1 and 2. For each vertex $x \in V(\Gamma)$, let

$$
\rho_{x}: M(\Gamma) \rightarrow\{x\}^{*}
$$

be the map which deletes all symbols other than $x$. For each pair of non-adjacent vertices $x$ and $y$, let

$$
\sigma_{x y}: M(\Gamma) \rightarrow\{x, y\}^{*}
$$

be the map which deletes all symbols other than $x$ and $y$. The following well-known proposition says that any two distinct elements of $M(\Gamma)$ are distinguished by at least one of the above morphisms; a proof can be found in [8].

Proposition 10. Let $u, v \in V(\Gamma)^{*}$ be words in the vertices of $\Gamma$, such that $\bar{u}$ and $\bar{v}$ are distinct elements of $M(\Gamma)$. Then either there exists a generator $x \in V(\Gamma)$ such that $\rho_{x}(u) \neq \rho_{x}(v)$ or there exist non-commuting generators $x, y \in V(\Gamma)$ such that $\sigma_{x y}(u) \neq \sigma_{x y}(v)$.

This result, while elementary, is a key tool in the theory of graph monoids, and it will be central to our proofs below. We note that Proposition 10 does not hold in the group case, with the obvious definitions of $\rho_{x}$ and $\sigma_{x y}$ as morphisms onto free groups of rank 1 and 2. For example, consider the graph $E_{1,1}$ with vertices $x$ of degree 0 and $y$ and $z$ of degree 1 . Then the word $x y x^{-1} z x y^{-1} x^{-1} z^{-1} \in U\left(E_{1,1}\right)^{*}$ does not represent the identity in $G\left(E_{1,1}\right)$, but is not distinguished from the identity by a projection onto 1 or 2 generators. In fact, Proposition 10 is a key reason why the theory of graph monoids is more straightforward than that of graph groups, and is why we obtain stronger results in the monoid case. One can formulate a related but more technical proposition concerning reduced words in the group generators 18. Proposition 1], but this does not seem to be helpful for our purposes.

We proceed with a lemma characterising words which commute in a graph monoid, in terms of the projections of the form $\sigma_{x y}$.

Lemma 11. Let $u$ and $v$ be words in the vertices of $\Gamma$. Then $\bar{u}$ and $\bar{v}$ commute in $M(\Gamma)$ if and only if for every pair of non-commuting vertices $x$ and $y$, there exists a word $w \in\{x, y\}^{*}$ which is not a proper power and integers $p, q \geq 0$ such that $\sigma_{x y}(u)=w^{p}$ and $\sigma_{x y}(v)=w^{q}$. 
Proof. Certainly for any words $u$ and $v$ and vertex $x$ we have $\rho_{x}(u v)=\rho_{x}(v u)$, so by Proposition 10 we see that $\bar{u}$ and $\bar{v}$ commute if and only if for every pair of noncommuting vertices $x$ and $y$ we have $\sigma_{x y}(u v)=\sigma_{x y}(v u)$. Now $\sigma_{x y}$ is a morphism, so this is true if and only if for every $x$ and $y$,

$$
\sigma_{x y}(u) \sigma_{x y}(v)=\sigma_{x y}(v) \sigma_{x y}(u),
$$

that is, if $\sigma_{x y}(u)$ and $\sigma_{x y}(v)$ commute in the free monoid. But clearly, this is the case if and only if $\sigma_{x y}(u)$ and $\sigma_{x y}(v)$ are powers of a common subword, which can be chosen not to be a proper power.

We need also the following lemma, which gives a necessary criterion for distinct elements to commute.

Lemma 12. Let $u, v \in V(\Gamma)^{*}$ be words in the vertices of $\Gamma$, such that $\bar{u}$ and $\bar{v}$ are distinct elements of $M(\Gamma)$. Suppose further that $\bar{u}$ and $\bar{v}$ commute in $M(\Gamma)$. Then there exists a generator $x$ such that $\rho_{x}(u) \neq \rho_{x}(v)$.

Proof. Suppose not. Then by Proposition 10, there exist non-commuting generators $x$ and $y$ such that $\sigma_{x y}(u) \neq \sigma_{x y}(v)$. But now by Lemma 11$]$ there exists a word $w \in\{x, y\}^{*}$ which is not a proper power and integers $p, q \geq 0$ such that $\sigma_{x y}(u)=w^{p}$ and $\sigma_{x y}(v)=w^{q}$. Moreover, since $\sigma_{x y}(u)$ and $\sigma_{x y}(v)$ are distinct, we must have $w$ non-empty and $p \neq q$. Since $w$ is non-empty, it must contain either an $x$ or a $y$. Suppose without loss of generality that it contains $k \geq 1$ occurrences of the letter $x$. Then $\sigma_{x y}(u)$ contains $k p$ occurrences of $x$, while $\sigma_{x y}(v)$ contains $k q$ occurrences of $x$. It follows that $u$ and $v$ contain $k p$ and $k q$ occurrences of $x$ respectively, so that $\rho_{x}(u) \neq \rho_{x}(v)$. This contradicts our supposition and hence completes the proof.

Lemma 13. Suppose $u, v \in V(\Gamma)^{*}$ are words in the vertices of $\Gamma$, such that $\bar{u}$ and $\bar{v}$ commute. Let $x \in V(\Gamma)$ be a vertex which occurs in $u$. Then either $x$ occurs in $v$, or $x$ commutes with every letter which occurs in $v$.

Proof. Suppose for a contradiction that $x$ does not occur in $v$, and does not commute with some letter $y$ which occurs in $v$. By Lemma 11] there exists a word $w \in F(x, y)$ and integers $p, q \geq 0$ such that $\sigma_{x y}(u)=w^{p}$ and $\sigma_{x y}(v)=w^{q}$. Now $u$ contains the letter $x$, so $\sigma_{x y}(u)=w^{p}$ contains the letter $x$, so $w$ must contain the letter $x$. On the other hand, $\sigma_{x y}(v)=w^{q}$ does not contain the letter $x$, so we must have $q=0$ and $\sigma_{x y}(v)=\epsilon$. But $v$ contains an occurrence of $y$, so $\sigma_{x y}$ contains an occurrence of $y$, and in particular is non-empty. This gives the required contradiction.

Lemma 14. Suppose $M(\Gamma)$ satisfies $\phi\left(\Omega \times \overline{E_{0,1}}\right)$. Then $\Gamma$ has an induced subgraph isomorphic to $\Gamma_{1} \times \overline{E_{0,1}}$ where $\Gamma_{1}$ satisfies $\phi(\Omega)$. Moreover, if $M\left(\Omega \times \overline{E_{0,1}}\right)$ embeds into $M(\Gamma)$ then $M(\Omega)$ embeds into $M\left(\Gamma_{1}\right)$.

Proof. Let $S$ be a subset of $M(\Gamma)$ with commutation graph isomorphic to $\Omega \times$ $\overline{E_{0,1}}$, let $e, e^{\prime} \in S$ be the elements which map to the vertices of $\overline{E_{0,1}}$ under this isomorphism, and let $S^{\prime}=S \backslash\left\{e, e^{\prime}\right\}$. Thus, $e$ and $e^{\prime}$ commute with every element of $S^{\prime}$, but not with each other.

Let $\Gamma_{1}$ be the subgraph induced by the set of all vertices in $V(\Gamma)$ which occur in the support of elements in $S^{\prime}$. It is immediate from the definition that $\Gamma_{1}$ satisfies $\phi(\Omega)$. Moreover, if $S$ actually generates a submonoid isomorphic to $M\left(\Omega \times \overline{E_{0,1}}\right)$ and $e$ and $e^{\prime}$ are chosen appropriately, then $M(\Omega)$ embeds in $M\left(\Gamma_{1}\right)$. 
By Lemma 11 we may choose vertices $y, z \in V(\Gamma)$ such that $\sigma_{y z}(e)$ and $\sigma_{y z}\left(e^{\prime}\right)$ are not powers of a common subword. Let $\Gamma_{2}$ be the subgraph induced by the vertex set $\{y, z\}$. To prove the lemma, it will suffice to show that $V\left(\Gamma_{1}\right)$ and $V\left(\Gamma_{2}\right)$ are disjoint, and that every vertex in $\Gamma_{2}$ is adjacent to every vertex in $\Gamma_{1}$.

First, we claim that $V\left(\Gamma_{1}\right)$ and $V\left(\Gamma_{2}\right)$ are disjoint, that is, that $y$ and $z$ do not lie in $V\left(\Gamma_{1}\right)$. Indeed suppose for a contradiction that at least one of them does, and let $d \in S^{\prime}$ be an element with support containing $y$ or $z$. Then $\sigma_{y z}(d)$ is non-empty. We know that $d$ commutes with $e$ and $e^{\prime}$, so by Lemma 11 we have $\sigma_{y z}(e)$ and $\sigma_{y z}(d)$ are powers of a common subword, and likewise that $\sigma_{y z}\left(e^{\prime}\right)$ and $\sigma_{y z}(d)$. But the relation of being powers of a common subword is transitive through non-empty words, so it would follow that $\sigma_{y z}(e)$ and $\sigma_{y z}\left(e^{\prime}\right)$ are powers of a common subword, giving the required contradiction.

Now since every element in $S^{\prime}$ commutes with $e$ and with $e^{\prime}$, Lemma 13 tells us that every letter in $\Gamma_{1}$ commutes with $y$ and with $z$. This completes the proof.

An inductive applications of Lemma 14 leads to the first main theorem of this section. We note that the case $j=1$ can also be obtained as a consequence of Theorem 7

Theorem 15. Let $j \geq 0$. Then $M(\Gamma)$ satisfies $\phi\left(\overline{E_{0, j}}\right)$ if and only if $\overline{E_{0, j}}$ embeds in $\Gamma$.

Proof. Suppose the direct implication is false, and let $j \geq 0$ be minimal such that there exists a graph $\Gamma$ such that $M(\Gamma)$ satisfies $\phi\left(\overline{E_{0, j}}\right)$ but $\overline{E_{0, j}}$ does not embed into $\Gamma$. Certainly $j \neq 0$, since $\overline{E_{0,0}}$ is the graph with no vertices, which certainly embeds into $\Gamma$.

Otherwise, we have $\overline{E_{0, j}}=\overline{E_{0, j-1}} \times \overline{E_{0,1}}$, so by Lemma 14 we see that $\Gamma$ has a subgraph isomorphic to $\Gamma_{1} \times \overline{E_{0,1}}$ where $\Gamma_{1}$ satisfies $\phi\left(\overline{E_{0, j}}\right)$. By the minimality assumption on $j, \overline{E_{0, j-1}}$ embeds into $\Gamma_{1}$, and it follows that $\overline{E_{0, j-1}} \times \overline{E_{0,1}}=\overline{E_{0, j}}$ embeds into $\Gamma_{1} \times \overline{E_{0,1}}$, and hence into $\Gamma$ as required.

The converse implication is immediate.

Before proving our second main theorem of this section, we need the following preliminary step. We remark that Batty and Goda 1] have observed that an analogous result holds for groups.

Proposition 16. Let $\Gamma$ be a graph not containing an induced subgraph isomorphic to the complete graph $\overline{E_{n, 0}}$ on $n$ vertices. Then $M(\Gamma)$ does not have a submonoid isomorphic to the free commutative monoid of rank $n$.

Proof. Suppose false for a contradiction, and let $\Gamma$ be a graph of minimal degree such that the claim fails. Let $u_{1}, \ldots u_{n} \in V(\Gamma)^{*}$ be words in the vertices of $\Gamma$ such that the corresponding elements $\overline{u_{1}}, \ldots, \overline{u_{n}} \in M(\Gamma)$ generate a free commutative monoid $N$ of rank $n$.

It follows from elementary linear algebra that the free commutative monoid of rank $n$ does not embed into a free commutative monoid of rank less than $n$, so we may assume that $\Gamma$ is not a complete graph and choose non-adjacent vertices $x, y \in V(\Gamma)$.

It follows from Lemma 11 that there exists a word $r \in\{x, y\}^{*}$ such that each $\sigma_{x y}\left(u_{i}\right)$ is of the form $r^{q}$ for some $q \geq 0$. Since $\Gamma$ is of minimal degree, every vertex of $\Gamma$ must occur in some $u_{i}$. In particular, $x$ and $y$ must each occur in some $u_{i}$ and so they must both occur in $r$. 
We define a morphism $f: M(\Gamma) \rightarrow M(\Gamma)$ by letting $f(w)$ be obtained from $w$ by deleting all occurrences of the generator $x$. We claim that this morphism is injective on $N$. Indeed, suppose $w, w^{\prime} \in V(\Omega)^{*}$ represent distinct elements of $N$. Then by Lemma 12 we have $\rho_{a}(w) \neq \rho_{a}\left(w^{\prime}\right)$ for some $a \in V(\Omega)$. We claim that we may assume without loss of generality that $a \neq x$. Indeed, by our observations above, $\sigma_{x y}(w)=r^{p}$ and $\sigma_{x y}\left(w^{\prime}\right)=r^{q}$ for some $p, q \geq 0$. If $\rho_{x}(w) \neq \rho_{x}\left(w^{\prime}\right)$ then we must have $p \neq q$. But since $r$ contains at least one occurrence of $y$, it follows that $\rho_{y}(w) \neq \rho_{y}\left(w^{\prime}\right)$ so we can instead take $a=y$.

Now we have

$$
\rho_{a}(f(w))=\rho_{a}(w) \neq \rho_{a}\left(w^{\prime}\right)=\rho_{a}\left(f\left(w^{\prime}\right)\right),
$$

so that $\overline{f(w)} \neq \overline{f\left(w^{\prime}\right)}$. This proves the claim that $f$ is injective.

Now since the image $f(N)$ is contained within the induced subgraph with vertex set $V(\Gamma) \backslash\{x\}$, this contradicts the minimality assumption on $\Gamma$ and completes the proof.

We are now ready to prove the following theorem.

Theorem 17. Let $i, j \geq 0$. Then $M(\Gamma)$ has a submonoid isomorphic to a direct product of $i$ rank 1 free monoids and $j$ non-abelian free monoids if and only if $\overline{E_{i, j}}$ embeds in $\Gamma$.

Proof. Suppose $M(\Gamma)$ has a submonoid isomorphic to a direct product of product of $i$ rank 1 free monoids and $j$ non-abelian free monoids. Then clearly, $M(\Gamma)$ has a submonoid isomorphic to $M\left(\overline{E_{i, j}}\right)$. Notice that

$$
\overline{E_{i, j}}=\overline{E_{i, 0}} \times \overline{E_{0, j}}=\overline{E_{i, 0}} \times \overline{E_{0,1}} \times \cdots \times \overline{E_{0,1}} .
$$

By an inductive application of Lemma 14 we deduce that $\Gamma$ has a subgraph isomorphic to $\Gamma_{1} \times \overline{E_{0, j}}$ where the free commutative monoid $M\left(\overline{E_{i, 0}}\right)$ of rank $i$ embeds in $M\left(\Gamma_{1}\right)$.

Now by Proposition [16 we deduce that $\Gamma_{1}$ contains a complete subgraph with $i$ vertices. It follows that $\Gamma_{1} \times \overline{E_{0, j}}$ has a induced subgraph isomorphic to $\overline{E_{i, j}}$, and hence so does $\Gamma$.

\section{Other Graph Monoids and Groups}

It seems natural to ask whether similar results hold for other graphs, that is, whether there are other graphs $\Gamma$ with the property that $G(\Omega)$ or $M(\Omega)$ satisfies $\phi(\Gamma)$ only when $\Gamma$ embeds in $\Omega$.

A related, but weaker, property has been considered by Batty and Goda [1. They call a graph group $G(\Gamma)$ unconcealable if it embeds into a graph group $G(\Omega)$ only when $\Gamma$ embeds into $\Omega$. They observe that the free group of rank 2 and all free abelian groups are unconcealable, and conjecture that direct products of free groups of rank 1 and 2 also have this property. Thus, our Corollary 9 proves one case of their conjecture; the general case remains open.

The notion of unconcealability applies equally naturally to monoids, and our Theorem [15] is the natural monoid-theoretic analogue of Batty and Goda's conjecture. In fact, in the monoid case, it transpires that this result is best possible, in the sense that all graphs not covered by that theorem admit concealments.

Proposition 18. If $M(\Gamma)$ is unconcealable then $\Gamma=\overline{E_{i, j}}$ for some $i, j \geq 0$. 
Proof. Suppose $M(\Gamma)$ is unconcealable, and consider the direct product of the projections $\sigma_{x y}$ and $\rho_{x}$. This is an injective (by Proposition 10) morphism from $M(\Gamma)$ to a direct product of free monoids of rank 1 and 2, that is, an embedding of $M(\Gamma)$ into a monoid of the form $M\left(\overline{E_{i^{\prime}, j^{\prime}}}\right)$. Since $M(\Gamma)$ is unconcealable, $\Gamma$ must embed into $\overline{E_{i^{\prime}, j^{\prime}}}$. It now follows easily that $\Gamma$ is of the form $\overline{E_{i, j}}$.

In the group case, the lack of a counterpart to Proposition 10 once again means that things are not so straightforward. In general, it is not clear exactly which graph groups are unconcealable or have our stronger property.

Recall that an assembly group is a graph group which can be built up from copies of $\mathbb{Z}$ using free and direct products. Droms, Servatius and Servatius 11] have shown that no non-assembly graph group embeds into an assembly group. They observe also that $G(\Gamma)$ (with $\Gamma$ finite) is an assembly group if and only if $\Gamma$ contains no embedded copy of $L_{3}$. Thus, their result can be interpreted as saying that $G\left(L_{3}\right)$ is unconcealable. It seems natural also to ask if this graph has our stronger property.

Question 19. Is there an assembly group satisfying $\phi\left(L_{3}\right)$ ?

The rest of this section is devoted to a combinatorial construction which yields a concealment for a large number of graph groups (and monoids). Specifically, we show that for $G(\Gamma)$ to be unconcealable it is necessary either that every vertex has degree $|\Gamma|-2$ or more (that is, $\Gamma=\overline{E_{i, j}}$ for some $i, j \geq 0$ ) or that $\Gamma$ has vertices of degree $|\Gamma|-2$ and $|\Gamma|-3$.

Let $\Gamma$ be a graph which does not satisfy this condition, that is, which has a vertex of degree $|\Gamma|-3$ or less, but does not have vertices of degree both $|\Gamma|-2$ and $|\Gamma|-3$. Let $e$ be a vertex of maximal degree amongst those vertices having degree $|\Gamma|-3$ or less, and let $f$ and $g$ be vertices which are not adjacent to $e$.

Let $e_{0}$ and $e_{1}$ be new symbols not in $V(\Gamma)$ and define a new graph $\Omega$ with

$$
\begin{gathered}
V(\Omega)=[V(\Gamma) \backslash\{e\}] \cup\left\{e_{0}, e_{1}\right\}, \text { and } \\
E(\Omega)=V(\Omega) \cup\left\{\left(e_{0}, a\right),\left(a, e_{0}\right),\left(a, e_{1}\right),\left(e_{1}, a\right) \mid(e, a) \in E(\Gamma)\right\} \\
\cup\left\{\left(e_{0}, f\right),\left(f, e_{0}\right),\left(e_{1}, g\right),\left(g, e_{1}\right)\right\} .
\end{gathered}
$$

We claim that $G(\Gamma)$ and $M(\Gamma)$ are concealed in $G(\Omega)$ and $M(\Omega)$ respectively. We begin by showing that $\Gamma$ is not an induced subgraph of $\Omega$.

Proposition 20. $\Gamma$ does not embed in $\Omega$.

Proof. Suppose for a contradiction that $\Omega$ has an induced subgraph $\Sigma$ which is isomorphic to $\Gamma$. Since $|\Gamma|=|\Omega|-1, \Sigma$ must be induced by deleting one vertex from $\Omega$; call this vertex $v$.

By construction, $\Omega$ has $|e|_{\Gamma}+2$ more edges than $\Gamma$. In order for $\Sigma$ to have the same number of edges as $\Gamma$, it must be that $|v|_{\Omega}=|e|_{\Gamma}+2$. In particular $v$ cannot be $e_{0}$ or $e_{1}$, both of which are constructed to have degree $|e|_{\Gamma}+1$ in $\Omega$. It follows that $v$ is a vertex from $\Gamma$. Now by the construction of $\Omega,|v|_{\Gamma}$ must be either $|v|_{\Omega}-1=|e|_{\Gamma}+1$ (if $v=f, v=g$ or $\left.(v, e) \in E(\Gamma)\right)$ or $|v|_{\Omega}=|e|_{\Gamma}+2$ (otherwise). Hence, by the maximality assumption on $|e|_{\Gamma}$, either $|v|_{\Gamma}=|\Gamma|-1$ or $|v|_{\Gamma}=|\Gamma|-2$.

Suppose first that $|v|_{\Gamma}=|\Gamma|-1$, that is, that $v$ is central in $\Gamma$. Note that $v$ cannot be $f$ or $g$, since neither commute with $e$ in $\Gamma$. Now suppose a vertex $x$ is central in $\Sigma$. Certainly $x \neq e_{0}$, since $e_{0}$ does not commute with $g$ in $\Omega$, and $g$ remains in the induced subgraph. By a symmetrical argument, $x \neq e_{1}$, so $x$ must 
also be a vertex in $\Gamma$. Moreover, $x$ commutes with every vertex in $\Sigma$ and also with $v$. It follows easily that $x$ is central in $\Gamma$. We have shown that every central vertex in $\Sigma$ is a central vertex in $\Gamma$, and we know also that $v$ is a central vertex in $\Gamma$. But now $\Sigma$ has strictly fewer central vertices than $\Gamma$, which contradicts the assumption that $\Sigma$ is isomorphic to $\Gamma$.

Now suppose that $|v|_{\Gamma}=|\Gamma|-2$. We have already seen that $|e|_{\Gamma}$ is either $|v|_{\Gamma}-1$ or $|v|_{\Gamma}-2$. But by our original assumptions, $\Gamma$ cannot contain a vertex of degree $|\Gamma|-3$, so it must be that $|e|_{\Gamma}=|\Gamma|-4$. It follows from the construction of $\Omega$ that $\Omega$ has the same number of vertices of degree greater than or equal to $|\Gamma|-2$ that $\Gamma$ does. But $v$ has degree $|\Gamma|-2$ and is missing from $\Sigma$. Hence, $\Sigma$ has strictly fewer vertices of degree greater than or equal to $|\Gamma|-2$ than $\Gamma$, which again gives the required contradiction.

Now considering for example the subset $(\Gamma \backslash\{e\}) \cup\left\{e_{0} e_{1} e_{0} e_{1}\right\}$ it is clear that $M(\Omega)$ has a subset with commutation graph $\Gamma$, and so both $M(\Omega)$ and $G(\Omega)$ satisfy $\phi(\Gamma)$. In fact, we can go further. Define a monoid morphism

$$
\tau: U(\Gamma)^{*} \rightarrow U(\Omega)^{*}, \quad \tau(x)= \begin{cases}e_{0} e_{1} e_{0} e_{1} & \text { if } x=e ; \text { or } \\ e_{1}^{-1} e_{0}^{-1} e_{1}^{-1} e_{0}^{-1} & \text { if } x=e^{-1} ; \text { or } \\ x & \text { if } x \in U(\Gamma) \backslash\left\{e, e^{-1}\right\} .\end{cases}
$$

It is immediate from the definition of $\Omega$ that $\tau$ respects the defining relations in $G(\Gamma)$, and so induces a well-defined morphism

$$
\bar{\tau}: G(\Gamma) \rightarrow G(\Omega), \quad \bar{w} \mapsto \overline{\tau(w)} .
$$

A straightforward but technical argument shows that $\bar{\tau}$ is injective, thus completing the proof of the following.

Theorem 21. Let $\Gamma$ be a graph which has a vertex of degree $|\Gamma|-3$ or less, but does not have both a vertex of degree $|\Gamma|-3$ and a vertex of degree $|\Gamma|-2$. Then there exists a graph $\Omega$ with $|\Omega|=|\Gamma|+1$ such that $G(\Gamma)$ embeds into $G(\Omega)$, but $\Gamma$ does not embed into $\Omega$.

\section{ACKNOWLEDGEMENTS}

The research documented here was started while the author was at Carleton University, supported by the Leverhulme Trust. It was completed, and this paper written, at Universität Kassel, where it was supported by a Marie Curie Intra-European Fellowship within the 6th European Community Framework Programme. The author would like to thank Mike Batty and Keith Goda for many helpful discussions. He would also like to thank Kirsty for all her support and encouragement.

\section{REFERENCES}

[1] M. Batty and K. M. Goda. private communication.

[2] M. Bestvina and N. Brady. Morse theory and finiteness properties of groups. Invent. Math., 129(3):445-470, 1997.

[3] N. Brady and J. Meier. Connectivity at infinity for right angled Artin groups. Trans. Amer. Math. Soc., 353(1):117-132, 2001.

[4] V. Bruyère and C. De Felice. Any lifting of a trace coding is a word coding. Inform. and Comput., 130(2):183-193, 1996.

[5] M. Chrobak and W. Rytter. Unique decipherability for partially commutative alphabets. Fund. Inform., 10(3):323-336, 1987. 
[6] M. W. Davis and T. Januszkiewicz. Right-angled Artin groups are commensurable with rightangled Coxeter groups. J. Pure Appl. Algebra, 153(3):229-235, 2000.

[7] W. Dicks and I. J. Leary. Presentations for subgroups of Artin groups. Proc. Amer. Math. Soc., 127(2):343-348, 1999.

[8] V. Diekert and Y. Métivier. Partial commutation and traces. In Handbook of Formal Languages, Vol. 3, pages 457-533. Springer, Berlin, 1997.

[9] V. Diekert and G. Rozenberg, editors. The Book of Traces. World Scientific Publishing Co. Inc., River Edge, NJ, 1995.

[10] C. Droms. Subgroups of graph groups. J. Algebra, 110(2):519-522, 1987.

[11] C. Droms, B. Servatius, and H. Servatius. Groups assembled from free and direct products. Discrete Math., 109(1-3):69-75, 1992.

[12] A. J. Duncan, I. V. Kazatchkov, and V. N. Remeslennikov. Centraliser dimension and universal classes of groups. arXiv:math.GR/0502498 2005.

[13] J. Howie. Bestvina-Brady groups and the plus construction. Math. Proc. Cambridge Philos. Soc., 127(3):487-493, 1999.

[14] I. Kapovich, R. Weidmann, and A. Myasnikov. Foldings, graphs of groups and the membership problem. Internat. J. Algebra Comput., 15(1):95-128, 2005.

[15] R. C. Lyndon and P. E. Schupp. Combinatorial Group Theory. Springer-Verlag, 1977.

[16] K. A. Mikhailova. The occurence problem for direct products of groups. Dokl. Akad. Nauk SSSR, 119:1103-1105, 1958. English translation in Soviet Math. Dokl. 70:201-204, 1966.

[17] E. Ochmański. On morphisms of trace monoids. In STACS 88 (Bordeaux, 1988), volume 294 of Lecture Notes in Comput. Sci., pages 346-355. Springer, Berlin, 1988.

[18] H. Servatius. Automorphisms of graph groups. J. Algebra, 126(1):34-60, 1989. 